\title{
SÍNTESIS Y CARACTERIZACIÓN DE LA MEZCLA POLIPROPILENO- POLIESTIRENO EXPANDIDO (ICOPOR) RECICLADO COMO ALTERNATIVA PARA EL PROCESO DE PRODUCCIÓN DE AUTOPARTES
}

\author{
Daylin Julieth Betancourt S. ${ }^{1}$ \\ Johanna Karina Solano M. $\stackrel{2}{2}$ \\ Recibido el 14 de junio de 2015, aprobado el 11 de noviembre de 2015 y \\ actualizado el 20 de mayo de 2016
}

DOI: 10.17151/luaz.2016.43.13

\section{RESUMEN}

En el proceso de fabricación de autopartes se incluye el polipropileno (PP) como materia prima principal y la fibra de vidrio como insumo para la producción de ventiladores utilizados en los sistemas de refrigeración de los automotores. Los estándares de fabricación deben garantizar la resistencia de los productos en condiciones a las que estarán sometidos dentro del motor. Para lograrlo, el polipropileno es mezclado con fibra de vidrio en el proceso de inyección para aumentar la resistencia y la dureza del producto final, debido a que está expuesto a cambios de temperatura, choques con otras piezas, desgaste e impactos inesperados en el vehículo. Este estudio sintetizó y caracterizó un material obtenido a partir de la mezcla polipropileno puro-poliestireno expandido (EPS) (icopor) reciclado como una alternativa de aprovechamiento para el icopor dentro del proceso de fabricación de autopartes a modo de sustituto de la fibra de vidrio en el proceso de producción, siguiendo pasos como el tamizaje de los tamaños de material, la mezcla de los mismos, la obtención de probetas y la realización de ensayos mecánicos y térmicos. El objetivo fue comparar las propiedades mecánicas y térmicas del material obtenido (PP-EPS) con el material actualmente utilizado (PP-fibra de vidrio) para el que no se tenían estudios previos y de esta forma determinar la viabilidad de incorporación de un residuo a un proceso 
productivo y evitar su disposición directa favoreciendo el reciclaje del mismo. Las mezclas se caracterizaron mediante ensayos de tensión-deformación, dureza Shore A y análisis termogravimétrico (TGA) de los cuales se obtuvo favorecimiento en las propiedades térmicas y reducción de la resistencia mecánica en el uso de icopor, resaltando por supuesto el ahorro significativo en el cambio de insumos del proceso productivo.

\title{
PALABRAS CLAVE
}

Aprovechamiento de residuos, residuos sólidos, TGA, poliestireno expandido, reciclaje.

\author{
SYNTHESIS AND CHARACTERIZATION OF EXPANDED MIXED \\ POLYSTYRENE - POLYPROPYLENE (STYROFOAM) RECYCLED AS AN \\ ALTERNATIVE FOR PRODUCTION PROCESS OF AUTOPARTS
}

\begin{abstract}
Polypropylene (PP) as main raw material and fiberglass as input for the production of fans used in the cooling systems of motor is included in the process of manufacturing parts. Manufacturing standards must guarantee the resistance of the product in conditions that will be subject within the engine. To achieve this, the polypropylene is mixed with fiberglass in the injection process to increase the strength and hardness of the final product, as it is exposed to temperature changes, impacts with other parts, wear and unexpected impacts on the vehicle. This study synthesized and characterized a material obtained from the pure-polypropylene blend expanded polystyrene (EPS) (polystyrene) recycling as an alternative use for polystyrene in the auto parts manufacturing process as a substitute for fiberglass production process, following steps as screening media sizes, the mixture thereof, and obtaining specimens performing mechanical and thermal tests. The aim was to compare the mechanical and thermal properties of the material obtained (PP-EPS) with the material currently used (PP-fiberglass) for which they had no previous
\end{abstract}


studies and thus determine the feasibility of incorporating a waste to a production in order to avoid direct disposal and thus promote the recycling process itself. The blends were characterized by stress-strain tests, Shore $A$ hardness and thermogravimetric analysis (TGA) of which was obtained favoring the thermal properties and mechanical strength reduction in the use of polystyrene, of course emphasizing the significant savings in change of inputs in the production process.

\section{KEY WORDS}

Waste utilization, solid waste, TGA, expanded polystyrene, recycling.

\section{INTRODUCCIÓN}

La industria de la transformación de materias primas plásticas es de constante movimiento debido a la gran demanda de la población por productos de bajo costo, especialmente por su característica de ser desechables. Asimismo, la cantidad de residuos generados es alta teniendo en cuenta que muchos de los productos como envases de alimentos o embalaje son instantáneamente desechados luego de consumir o utilizar su contenido. Ambiental y económicamente el EPS es un polímero de poco aprovechamiento y de difícil disposición final por su baja compresibilidad para ser llevado a un relleno sanitario como sucede en la ciudad de Bogotá (Contreras, 2006). En la mayoría de los casos, reutilizarlo o reciclarlo económicamente no es viable porque su baja densidad lo hace voluminoso y liviano y no representa oportunidad de negocio para comprarlo por peso.

En Colombia se ha impulsado el nivel de producción y consumo de productos transformados de plásticos debido a que es un componente transversal a diversas industrias (Contreras, 2014). Aunque el país no sea gran productor de las materias primas, ha crecido fuertemente en la industria de la transformación de estas 
resinas, ubicando en Bogotá el 55\% de las empresas dedicadas a estas actividades (MinCIT, 2013). No obstante, de las 859.000 toneladas de plástico desechado en el año, solo es reciclado el 28\% (CEMPRE Colombia, 2013), equivalente a 240.520 toneladas y dentro de las cuales solo 500 toneladas son de icopor, participando activamente con el $0,2 \%$ del total de reciclaje de plásticos en el país (Fundación Verde Natura, 2015).

Las investigaciones que motivaron este estudio incluyen el icopor como aditivo para la mejora de mezclas asfálticas hasta de $50 \%$ en cuanto a resistencia (Cárdenas \& Fonseca, 2009), además de la mejora de residuos de madera para la elaboración de muebles en Brasil, que plantea un proceso mecánico de reciclaje de poliestireno expandido para aumentar la resistencia de la madera y como agente de acoplamiento para la misma (Matheus, Dettenborn, Zeni \& Zattera, 2011). La alternativa de aprovechamiento del residuo presentada en este documento posibilita la valorización del mismo, ya que se caracterizó un material compuesto por materia prima de primera mano y material reciclado, para este caso el polipropileno de primera y poliestireno expandido EPS (icopor) reciclado de los residuos de embalaje de electrodomésticos.

\section{EXPERIMENTACIÓN}

La metodología se basó en la fabricación de muestras piloto para llevar a cabo ensayos con el material de PP+EPS reciclado en el proceso de inyección, teniendo en cuenta las variables propuestas y la implementación de un molde para obtener las probetas normalizadas y llevar a cabo las pruebas térmicas y mecánicas. Los ensayos llevados a cabo correspondieron a tensión-deformación bajo la norma ASTM D638-02a, dureza Shore A y TGA para la mezcla propuesta, la mezcla original y el polipropileno puro con el fin de establecer comparaciones entre las propiedades de cada uno. 


\section{Equipos y materiales}

Para el proceso de fabricación de autopartes plásticas se utilizó una máquina inyectora. Su sistema requiere que el material ingrese en la tolva de forma granulada para fundirlo rápidamente y dirigirlo por un tornillo sin fin hacia un molde metálico, en este caso, con la forma que requiere la probeta para realizar los ensayos. También se requirió un equipo para reducir el tamaño de partícula del icopor reciclado, en este caso un molino de cuchillas ideal para la molienda de materiales blandos y semiduros (FRITSCH, 2014).

Dentro de los materiales, el icopor se recolectó de los residuos sólidos urbanos y se realizó una reducción de tamaño de partícula en el molino para introducirlo a la inyectora. Por su parte, el PP virgen y la fibra de vidrio solo fueron mezclados entre sí y el PP con el EPS reciclado para obtener las probetas finales.

\section{Síntesis del material}

Las variables seleccionadas fueron el tamaño de partícula y la composición de la mezcla (relación EPS/PP) debido a que el blanco de muestra (PP+fibra de vidrio) es dependiente de estas características.

El tamaño de partícula del icopor fue modificado contando con que el polipropileno por elección del fabricante tiene definido su tamaño en 3,2 $\mathrm{mm}$ para cada gránulo. La referencia específica del material PP corresponde a homopolímero con índice de fluidez de $40 \mathrm{~g} / 10 \mathrm{~min}$. El molino de cuchillas arroja tamaños de partícula superiores a $3 \mathrm{~mm}$, para lo cual se emplearon mallas que permitieron tamizar el material y obtener tres rangos de tamaño deseado que sigan indicaciones del diseño experimental para el poliestireno expandido.

Por su parte, la fibra de vidrio en pellets tiene una longitud de $3,5 \mathrm{~mm}$ por diseño del fabricante. 
En cuanto a la composición de la mezcla, habitualmente es 70\% PP y 30\% fibra de vidrio y fue utilizada como blanco de muestra. En base a ella fueron diseñados los experimentos de polipropileno y poliestireno expandido, de tal manera que se modificó la composición a partir de esta proporción.

Se utilizó un diseño experimental factorial, manejando dos variables y tres niveles para cada una de ellas obteniendo así 8 de cada una. Cada uno de los 8 ensayos fue triplicado por recomendación de la norma técnica ASTM D638-02 en un total de 24 ensayos para la prueba de tensión-deformación. La prueba de TGA fue aplicada a todas las variables una sola vez con un total de 8 muestras como se describe en la Tabla 1.

Tabla 1. Descripción del diseño de experimentos

\begin{tabular}{|c|c|c|c|}
\hline Variable & Ensayo & Especificación & Descripción \\
\hline \multirow{4}{*}{$\begin{array}{c}\text { Tamaño de } \\
\text { partícula }\end{array}$} & \multirow{4}{*}{$\begin{array}{c}\text { Tensión-deformación } \\
\text { Dureza } \\
\text { TGA }\end{array}$} & EPS pequeño & $\begin{array}{l}\text { PP } 3,2 \mathrm{~mm} \\
\text { EPS } 15 \mathrm{~mm}\end{array}$ \\
\hline & & EPS mediano & $\begin{array}{l}\text { PP } 3,2 \mathrm{~mm} \\
\text { EPS } 40 \mathrm{~mm}\end{array}$ \\
\hline & & EPS grande & $\begin{array}{l}\text { PP } 3,2 \mathrm{~mm} \\
\text { EPS } 90 \mathrm{~mm}\end{array}$ \\
\hline & & $\begin{array}{c}\text { Blanco de } \\
\text { muestra }\end{array}$ & $\begin{array}{c}\text { PP } 3,2 \mathrm{~mm} \\
\text { Fibra de vidrio } 3,5 \mathrm{~mm}\end{array}$ \\
\hline \multirow{4}{*}{$\begin{array}{l}\text { Composición } \\
\text { de la mezcla }\end{array}$} & \multirow{4}{*}{$\begin{array}{c}\text { Tensión-deformación } \\
\text { Dureza } \\
\text { TGA }\end{array}$} & $\%$ EPS $>\%$ PP & $\begin{array}{l}\text { EPS } 70 \% \\
\text { PP 30\% }\end{array}$ \\
\hline & & $\%$ EPS $<\%$ PP & $\begin{array}{l}\text { EPS } 30 \% \\
\text { PP } 70 \%\end{array}$ \\
\hline & & $\%$ EPS $=\% \mathrm{PP}$ & $\begin{array}{l}\text { PP } 50 \% \\
\text { EPS } 50 \%\end{array}$ \\
\hline & & $\begin{array}{c}\text { Blanco de } \\
\text { muestra }\end{array}$ & $\begin{array}{c}\text { PP } 70 \% \\
\text { Fibra de vidrio } 30 \%\end{array}$ \\
\hline
\end{tabular}




\section{Caracterización}

Los ensayos llevados a cabo corresponden a pruebas mecánicas y térmicas para caracterizar los materiales.

\section{Tensión-deformación}

Fueron necesarias 24 probetas con las que se recurrió a la Máquina de Ensayo Universal Ez-Lx tipo Shimadzu. El ensayo se realizó con base al método normalizado según la ASTM D638-02a. Se acondicionaron las probetas a $23 \pm 2^{\circ} \mathrm{C}$ y humedad relativa del $50 \pm 5 \%$ mínimo por 40 horas anteriores a la prueba. Se aplicaron las tolerancias de $\pm 1^{\circ} \mathrm{C}$ y humedad relativa de $\pm 2 \%$. Los resultados obtenidos de este ensayo correspondieron a tensión-deformación y módulo de elasticidad de los materiales.

En la Tabla 2 se presentan las ecuaciones necesarias para calcular el módulo de elasticidad E a partir del diagrama tensión-deformación de los materiales. Los valores de las variables en las ecuaciones involucradas fueron arrojados por el software Trapecio (Shimadzu) del equipo, entre ellas la fuerza aplicada y la longitud. El área, por el contrario, se obtuvo de la sección rectangular del centro de la probeta sumando el estiramiento unitario que sufrió. 
Tabla 2. Ecuaciones correspondientes al módulo de elasticidad

\begin{tabular}{|l|c|l|}
\hline Deformación unitaria & $\varepsilon=\frac{\delta}{l}$ & $\begin{array}{l}\delta=\text { Deformación } \\
l=\text { longitud }\end{array}$ \\
\hline Esfuerzo & $\sigma=\frac{F}{A}$ & $\begin{array}{l}F=\text { Fuerza aplicada } \\
A=\text { Área de la sección }\end{array}$ \\
\hline Módulo de elasticidad & $E=\frac{\sigma}{\varepsilon}$ & $\begin{array}{l}\sigma=\text { Esfuerzo } \\
\varepsilon=\text { Deformación unitaria }\end{array}$ \\
\hline
\end{tabular}

Fuente: Budynas \& Nisbett (2011).

\section{Dureza Shore A}

Se tomaron las muestras por triplicado al igual que el ensayo de tensióndeformación con 24 muestras en total. Para tratar los resultados fue necesario promediar los totales de las muestras y determinar la desviación estándar. El ángulo de penetración del durómetro es de $35^{\circ}$ sobre el material. Se eligió para el tratamiento de datos la combinación más adecuada con el valor más alto si lo que se desea es saber qué material es más duro o resistente a la penetración y/o rayaduras. Se utilizó el equipo DFH-100 rango Sensor Dual Fricción / carga 1,0 a $100 \mathrm{~kg}$, soportado en la norma ASTM D785-08. El quipo cuenta con un indentador de bola de acero, montado bajo un sensor de fuerza $100 \mathrm{~kg}$ sobre un carro posicionamiento vertical accionado por un motor. La muestra se montó sobre una mesa estacionaria directamente bajo el indentador. En seguida, un carro presionó hacia abajo el penetrador contra la muestra para aplicar un preliminar de carga de $10 \mathrm{~kg}$ durante 10 segundos. El carro aumentó la carga a un total de $100 \mathrm{~kg}$ durante 15 segundos.

\section{Análisis termogravimétrico (TGA)}


El equipo empleado para el ensayo de las 8 muestras de termogravimetría corresponde a un Analizador Termogravimétrico TGA/DSC1 Mettler Toledo guiado por la norma ASTM D6370-99. La muestra de $10 \mathrm{mg}$ fue colocada en la platina del analizador calibrado y se purgó el equipo a $75 \mathrm{~cm} 3 / \mathrm{min}$ con argón. La muestra se calentó por 2 minutos a $50^{\circ} \mathrm{C}$, en seguida se inició el calentamiento a razón de $10 \mathrm{C} \%$ min de $50^{\circ} \mathrm{C}$ a $560^{\circ} \mathrm{C}$, se dejó enfriar a $300^{\circ} \mathrm{C}$ y por 2 minutos se buscó el equilibrio de temperatura. Se cambia el gas de purga por aire con flujo de 75 $\mathrm{cm} 3 / \mathrm{min}$ y se aumenta la temperatura a razón de $10 \mathrm{C} / \mathrm{min}$ desde $300^{\circ} \mathrm{C}$ a $800^{\circ} \mathrm{C}$.

\section{RESULTADOS Y ANÁLISIS}

El tamaño de partícula del EPS fue indiferente al tamaño de partícula de la fibra de vidrio, porque con el uso de tamaños pequeños se generó oscurecimiento del material por la elevada temperatura que alcanza la inyectora para fundir el PP, por ende se prefieren tamaños superiores a $15 \mathrm{~mm}$.

Los datos elegidos para el análisis fueron los que registraron mejores resultados de cada prueba respecto al blanco de muestra en cada ítem como describen las tablas.

\section{Ensayo de tensión-deformación}


Tabla 3. Tensión-deformación de acuerdo al tamaño de partícula

\begin{tabular}{|c|c|c|}
\hline Tamaño & $\begin{array}{c}\text { Tensión } \\
\text { (MPa) }\end{array}$ & $\begin{array}{c}\text { Deformación } \\
\mathbf{( \% )}\end{array}$ \\
\hline Pequeño $(15 \mathrm{~mm})$ & 14,3 & $1,64 \pm 0,00$ \\
\hline Mediano $(\mathbf{4 0} \mathbf{~ m m})$ & $\mathbf{2 6 , 5}$ & $\mathbf{4 , 2 8} \pm \mathbf{0 , 0 1}$ \\
\hline Grande $(90 \mathrm{~mm})$ & 24,0 & $3,72 \pm 0,01$ \\
\hline Polipropileno puro & 39,0 & $4,98 \pm 0,02$ \\
\hline Polipropileno reforzado & 41,3 & $4,45 \pm 0,00$ \\
\hline
\end{tabular}

En la Tabla 3 se observa que el tamaño de $40 \mathrm{~mm}$ es el que más resistió tensión con un valor de $26 \mathrm{MPa}$ frente a los demás. La Figura 1 muestra el diagrama tensión-deformación para la combinación de material con EPS mediano frente a los datos obtenidos para el PP puro y el PP reforzado con fibra de vidrio.

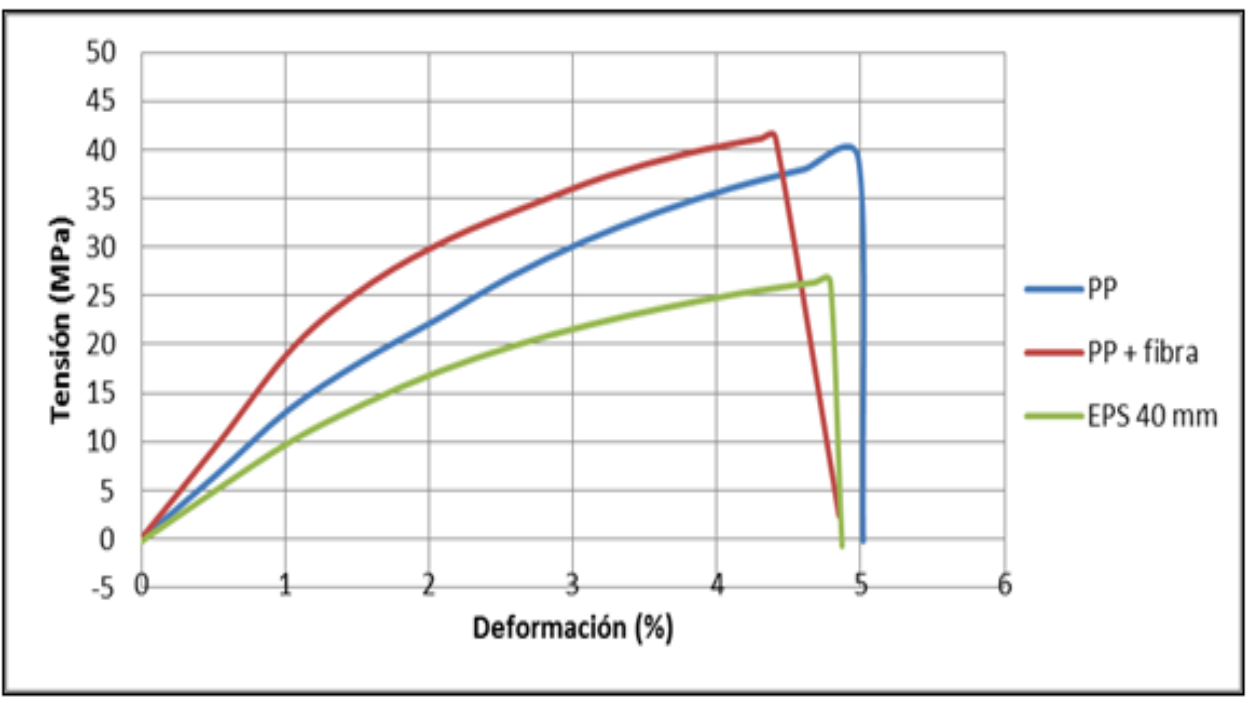

Figura 1. Curvas de tensión-deformación de acuerdo al tamaño de partícula. 
En los resultados obtenidos en el ensayo de tensión-deformación para esta combinación de material, se observa que es necesario estudiar a profundidad la mejora de las propiedades del PP respecto al tamaño de partícula del EPS, porque fue notorio que incluso el PP puro tiene mejores propiedades de resistencia. Es posible que el tamaño de partícula sea indiferente a los resultados de resistencia mecánica porque finalmente el material ingresa fundido al molde de inyección.

Fue relevante percibir que el PP con fibra de vidrio no mejora significativamente como lo plantea la empresa, pues los resultados muestran que el PP puro registra 39,05 MPa y con fibra 41,3 MPa.

Tabla 4. Tensión-deformación de acuerdo a la composición de la mezcla

\begin{tabular}{|c|c|c|}
\hline Composición & $\begin{array}{c}\text { Tensión } \\
\text { (MPa) }\end{array}$ & $\begin{array}{c}\text { Deformación } \\
\mathbf{( \% )}\end{array}$ \\
\hline 30 EPS/70 PP & 25,9 & $10,03 \pm 0,04$ \\
\hline $\mathbf{5 0 ~ E P S / 5 0 ~ P P ~}$ & $\mathbf{2 8 , 3}$ & $\mathbf{5 , 2 1 \pm 0 , 0 1}$ \\
\hline 70 EPS/30 PP & 14,3 & $2,53 \pm 0,01$ \\
\hline Polipropileno puro & 39,0 & $4,98 \pm 0,02$ \\
\hline Polipropileno reforzado & 41,3 & $4,45 \pm 0,00$ \\
\hline
\end{tabular}

De la misma forma que para el tamaño de partícula, fue la combinación intermedia la que se hace más ideal. Con una tensión máxima de 28,3 MPa, la composición

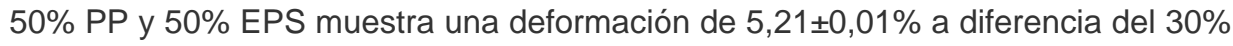
EPS y $70 \%$ PP que con solo $25,9 \mathrm{MPa}$ de tensión se ha deformado en un $10,03 \pm 0,04 \%$.

La Figura 2 corresponde al diagrama tensión-deformación para la variable de composición de la mezcla, donde se observa que el PP reforzado con fibra e incluso el PP puro tienen mayor resistencia a la tracción que la mezcla de PP con EPS al $50 \%$. 
En este caso, el EPS tampoco funcionó como aditivo apropiado para mejorar la calidad del PP respecto a la tensión- deformación, pues de la misma manera que con el tamaño de partícula, arrojó una tensión inferior que el PP puro y por supuesto menos aún que el PP reforzado con fibra de vidrio. El fenómeno puede ser explicado a partir de una posible incompatibilidad de los dos materiales para la distribución uniforme de las tensiones a las que es sometida la mezcla PP+EPS reciclado (Matheus et al., 2011).

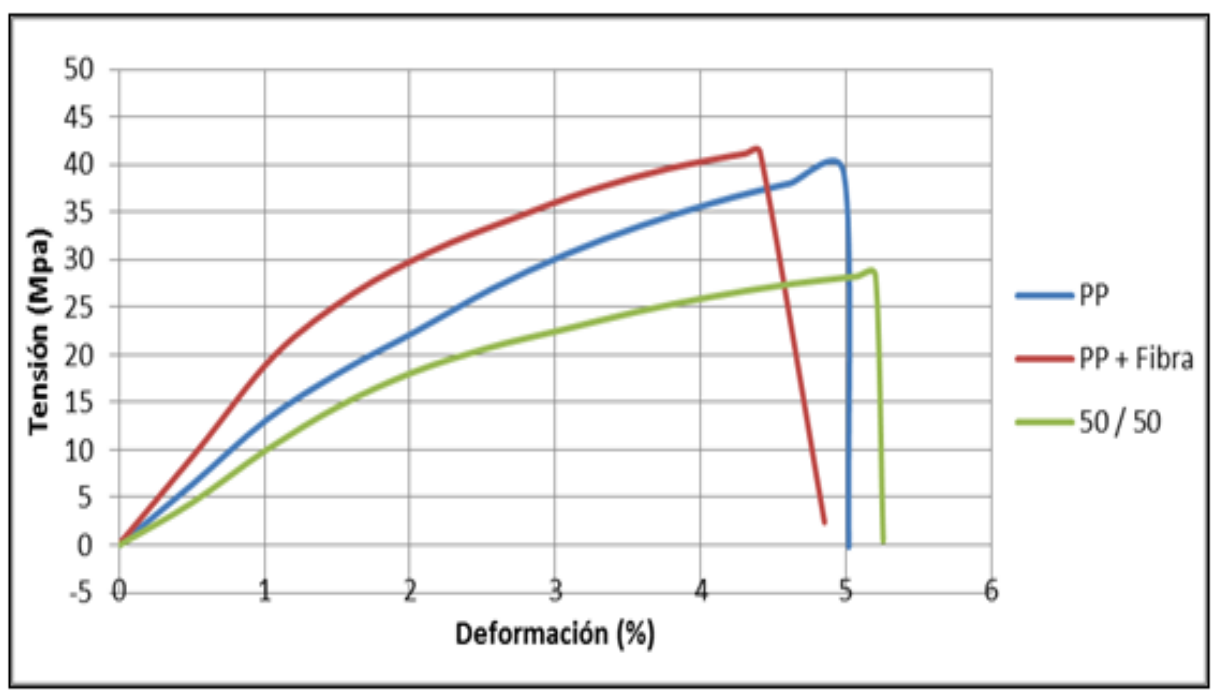

Figura 2. Curvas de tensión-deformación de acuerdo a la composición de la mezcla.

Los resultados obtenidos fueron más adecuados para unas pruebas que para otras según las combinaciones de las mezclas, las propiedades evaluadas y las variables trabajadas. Necesariamente hay que encontrar mediante herramientas estadísticas las combinaciones más adecuadas de material respecto a las variables. Se recomienda mantener las pruebas con la variable de composición de la mezcla mas no para tamaños de partícula, pues esta no arroja diferencias significativas debido a que finalmente en la inyectora se funde el material para 
llenado de moldes sin discriminar el tamaño que ingresó por la tolva de alimentación.

\section{Módulo de elasticidad}

De la misma forma que en la tensión de ruptura, fueron calculados los módulos de elasticidad para el tamaño de partícula y la composición de la mezcla comparados con el PP puro y el PP reforzado con fibra de vidrio, esta propiedad mecánica dio cuenta de la rigidez del material, pues a mayor módulo, mayor fue la rigidez.

Tabla 5. Resultados módulo de elasticidad de acuerdo al tamaño de partícula

\begin{tabular}{|c|c|}
\hline Tamaño & $\begin{array}{c}\text { Módulo de elasticidad } \\
\text { (GPa) }\end{array}$ \\
\hline Pequeño $(15 \mathrm{~mm})$ & $2,31 \pm 0,36$ \\
\hline Mediano $(40 \mathrm{~mm})$ & $3,12 \pm 0,81$ \\
\hline Grande $(90 \mathrm{~mm})$ & $3,21 \pm 1,62$ \\
\hline Polipropileno puro & $3,83 \pm 1,85$ \\
\hline Polipropileno reforzado & $4,00 \pm 1,61$ \\
\hline
\end{tabular}

El módulo de elasticidad tuvo una mejora significativa cuando el PP es reforzado con fibra de vidrio e incluso cuando es puro, pero en lo que respecta a la mezcla con EPS, la propiedad mostró una reducción cuando el tamaño de este disminuyó. Se encontró que el PP reforzado tiene el mayor módulo de elasticidad en comparación con el PP puro y el PP con EPS. El aumento de módulo de elasticidad está directamente relacionado con el aumento del tamaño de EPS, posiblemente igual que como funciona con la harina de madera en la elaboración de muebles, donde el EPS aporta capacidad de refuerzo y logró un módulo de elasticidad del doble al inicial (Bengtsson, Le Baillif \& Oksman, 2007). 
En este caso, el polipropileno tiene un módulo mayor que el EPS reciclado con $3,83 \pm 1,85 \mathrm{GPa}$ y el EPS oscila entre 0,0015 y 0,01 GPa (ANAPE, 2011), la diferencia significativa en la magnitud puede ocasionar la reducción del módulo de elasticidad de la mezcla con EPS reciclado.

Tabla 6. Resultados módulo de elasticidad de acuerdo a la composición de la mezcla

\begin{tabular}{|c|c|}
\hline Composición & $\begin{array}{c}\text { Módulo de elasticidad } \\
\text { (GPa) }\end{array}$ \\
\hline $\mathbf{3 0 ~ E P S / 7 0 ~ P P ~}$ & $\mathbf{3 , 5 3} \pm 1,16$ \\
\hline $50 \mathrm{EPS} / 50 \mathrm{PP}$ & $3,45 \pm \mathbf{1 , 5 7}$ \\
\hline $70 \mathrm{EPS} / 30 \mathrm{PP}$ & $3,38 \pm 1,73$ \\
\hline Polipropileno puro & $3,83 \pm 1,85$ \\
\hline Polipropileno reforzado & $4,00 \pm 1,61$ \\
\hline
\end{tabular}

Para la variable de composición de la mezcla, los resultados fueron similares a los de tamaño de partícula aunque se acercaron más a los de PP puro. No obstante, se observó que entre menor fue la cantidad del icopor en la mezcla, mejores fueron las propiedades de esta.

La combinación de mezcla propuesta más rígida fue de 30\% EPS reciclado y $70 \%$ PP para tener un valor de rigidez de $3,53 \pm 1,16 \mathrm{GPa}$. Es posible que para este tipo de polipropileno el módulo de elasticidad sea más alto, pero teóricamente este valor corresponde a $13 \mathrm{GPa}$ (Plasticbages Industrial, 2009), lo que mostró que posiblemente el EPS reciclado sí podría aumentar estas propiedades.

\section{Ensayo de dureza Shore A}

Las tablas 7 y 8 ilustran los resultados de dureza Shore tipo A para las combinaciones de material según las variables elegidas. 
Tabla 7. Resultados dureza Shore de acuerdo al tamaño de partícula

\begin{tabular}{|c|c|}
\hline Tamaño & Dureza \\
\hline Pequeño $(15 \mathrm{~mm})$ & $89 \pm 6$ \\
\hline Mediano $(40 \mathrm{~mm})$ & $90 \pm 6$ \\
\hline Grande $(90 \mathrm{~mm})$ & $93 \pm 4$ \\
\hline Polipropileno puro & $92 \pm 4$ \\
\hline Polipropileno reforzado & $94 \pm 4$ \\
\hline
\end{tabular}

El material con la dureza similar al PP es el de tamaño grande, correspondiente a $93 \pm 4$ en la escala Shore, esto indicó que el material no fue atravesado con facilidad. EI PP puro presentó un valor inferior a la mezcla con icopor grande e igual a la mezcla con icopor mediano. Sin embargo, el polipropileno reforzado con fibra siguió siendo superior en este valor con magnitud de $94 \pm 4$ en la escala.

Tabla 8. Resultados dureza de acuerdo a la composición de la mezcla

\begin{tabular}{|c|c|}
\hline Composición & Dureza \\
\hline $30 \mathrm{EPS} / 70 \mathrm{PP}$ & $90 \pm 5$ \\
\hline $50 \mathrm{EPS} / 50 \mathrm{PP}$ & $92 \pm 5$ \\
\hline $\mathbf{7 0} \mathrm{EPS} / 30 \mathrm{PP}$ & $\mathbf{9 3 \pm 4}$ \\
\hline Polipropileno puro & $92 \pm 4$ \\
\hline Polipropileno reforzado & $94 \pm 4$ \\
\hline
\end{tabular}


Las propiedades del material para la composición de la mezcla fueron similares a las del tamaño de partícula, mejoraron el PP puro pero el PP reforzado con fibra siguió siendo superior en una unidad de la escala.

La exposición de los materiales ante el penetrador del durómetro no hizo mayor daño a la forma del material en el intento de atravesarlo. Realmente fueron valores muy similares entre la mezcla original y la mezcla propuesta, para la que se recomienda seguir investigando sobre la combinación más adecuada con EPS, ya que se sabe que si mejora la dureza del PP como lo hace con los asfaltos y los residuos de madera, pues podría mejorar otro tipo de características.

En el caso específico del asfalto, el aporte del EPS reciclado no solo es en cuanto a dureza y resistencia, implícito a estas propiedades, hay una disminución de la susceptibilidad térmica del material, aumento de la estabilidad de la mezcla y, además, una reducción en su peso generando un aumento de vacíos y, por tanto, un espacio adicional entre las partículas para su reacomodación ante adversidades (Figueroa Infante, Reyes Lizcano, Hernández Barrera, Jiménez \& Bohórquez, 2007).

\section{Análisis termogravimétrico (TGA)}

Las tablas 9 y 10 muestran los resultados generales del ensayo de TGA para las dos variables estudiadas. Se tomaron en cuenta los valores cada $100^{\circ} \mathrm{C}$ para cada material y finalmente se calculó el porcentaje de masa inicial que queda luego de someter las muestras a $600^{\circ} \mathrm{C}$. Los valores ilustrados en la Tabla 9 corresponden al porcentaje de masa presente en la muestra aún. 
Tabla 9. Resultados termogravimetría de acuerdo al tamaño de partícula

\begin{tabular}{|c|c|c|c|c|c|}
\hline$\%$ masa $>$ Material & $\begin{array}{l}\text { Pequeño } \\
\text { (15 mm) }\end{array}$ & $\begin{array}{l}\text { Mediano } \\
(40 \mathrm{~mm})\end{array}$ & $\begin{array}{l}\text { Grande } \\
(90 \mathrm{~mm})\end{array}$ & PP puro & PP+fibra \\
\hline Inicial & 100 & 100 & 100 & 100 & 100 \\
\hline $\mathrm{A} 100^{\circ} \mathrm{C}$ & 99,93 & 99,95 & 99,92 & 100 & 100 \\
\hline $\mathrm{A} 200^{\circ} \mathrm{C}$ & 99,86 & 99,91 & 99,85 & 99,96 & 99,97 \\
\hline A $300^{\circ} \mathrm{C}$ & 97,36 & 97,32 & 97,22 & 98 & 97,57 \\
\hline $\mathrm{A} 400^{\circ} \mathrm{C}$ & 36,86 & 36,74 & 33,86 & 36,62 & 38,72 \\
\hline A $500^{\circ} \mathrm{C}$ & 1,3 & 0,98 & 0,17 & 8,81 & 0,92 \\
\hline $\mathrm{A} 600^{\circ} \mathrm{C}$ & 1,2 & 0,94 & 0,13 & 8,75 & 0,88 \\
\hline Masa inicial (g) & 14,744 & 15,711 & 16,287 & 17,454 & 18,372 \\
\hline Masa final $(\mathrm{g})$ & 0,012 & 0,0094 & 0,0013 & 0,0875 & 0,0088 \\
\hline
\end{tabular}

Los resultados consignados en la Tabla 9 muestran que el PP con EPS pequeño fue la mejor combinación con un porcentaje de masa inicial de 1,2\%, aún por debajo del PP puro con $8,75 \%$ de la masa inicial.

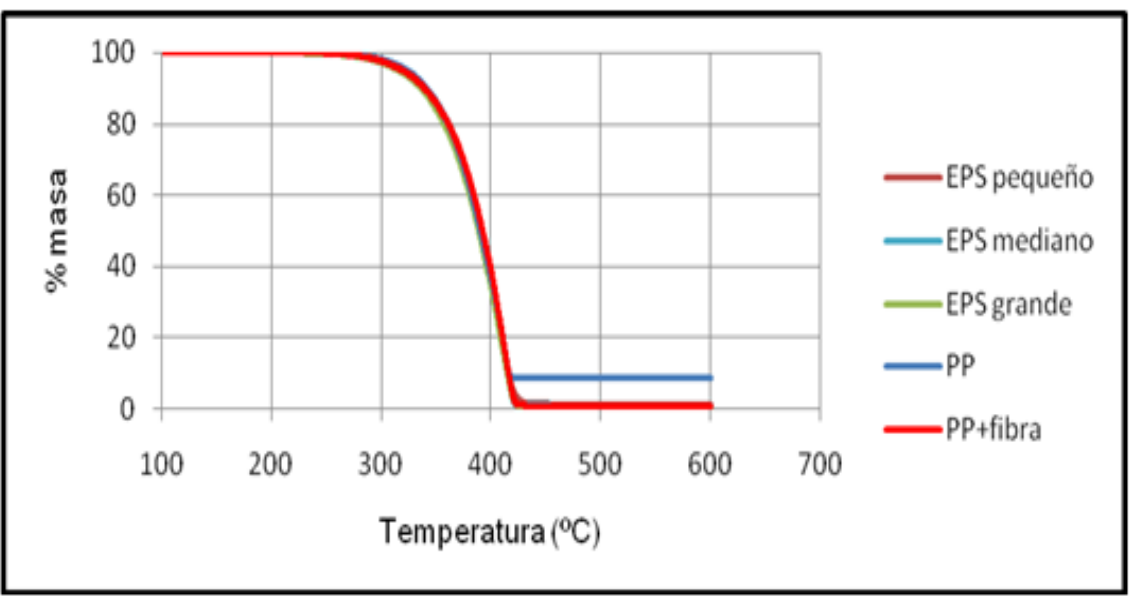

Figura 3. Termograma correspondiente al tamaño de partícula. 
El termograma muestra que todos los materiales tienen pérdidas significativas después de los $300^{\circ} \mathrm{C}$, pues al alcanzar los $400^{\circ} \mathrm{C}$ en todos quedó menos del $40 \%$ de la masa inicial. Fue notorio que aunque la mayor parte del tiempo el PP reforzado resistió más a los cambios de temperatura, luego de los $400^{\circ} \mathrm{C}$ tuvo una degradación más rápida que los demás materiales donde el PP puro perdió menos porcentaje de masa que si se combina bien sea con fibra de vidrio o EPS.

La literatura de soporte muestra que en las pruebas térmicas el polipropileno puro se comporta siempre de la misma manera sin importar la concentración que se maneje. Luego de los $400^{\circ} \mathrm{C}$ inicia la reducción acelerada de masa y finaliza con una cantidad cercana al $10 \%$, el fenómeno puede ser explicado desde la perspectiva de la homogeneidad de la mezcla, debido a que no son completamente compatibles y para ciertas propiedades de los materiales son independientes, lo que se refleja en que cada material toma su curva de temperatura vs. masa independientemente (Valea, Juanes, Miguez \& González, 2008).

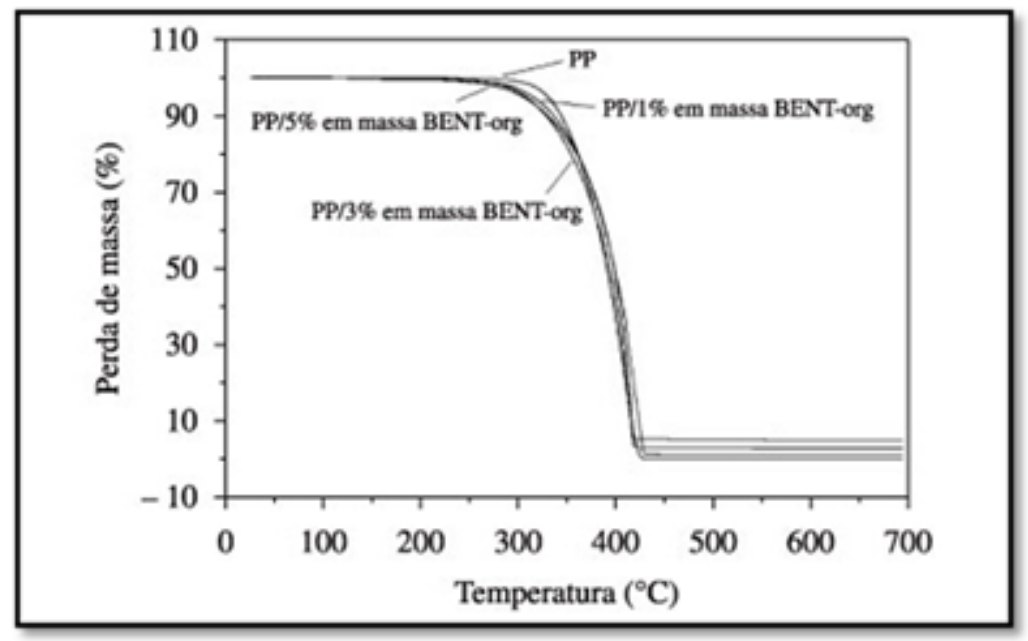

Fuente: Líbano, Visconte \& Pacheco (2012).

Figura 4. Termograma correspondiente al polipropileno puro. 
Tabla 10. Resultados termogravimetría de acuerdo a composición de la mezcla

\begin{tabular}{|c|c|c|c|c|c|}
\hline$\%$ masa Material & $30 \mathrm{EPS} / 70 \mathrm{PP}$ & $50 \mathrm{EPS} / 50 \mathrm{PP}$ & 70 EPS/30 PP & PP puro & $\mathrm{PP}+\mathrm{fibra}$ \\
\hline Inicial & 100 & 100 & 100 & 100 & 100 \\
\hline $\mathrm{A} 100^{\circ} \mathrm{C}$ & 100 & 100 & 100 & 100 & 100 \\
\hline $\mathrm{A} 200^{\circ} \mathrm{C}$ & 99,98 & 100 & 100 & 99,96 & 99,97 \\
\hline A $300^{\circ} \mathrm{C}$ & 97,32 & 96,78 & 97,61 & 98 & 97,57 \\
\hline $\mathrm{A} 400^{\circ} \mathrm{C}$ & 25,13 & 25,99 & 41,28 & 36,62 & 38,72 \\
\hline A $500^{\circ} \mathrm{C}$ & 0,25 & 0,54 & 5,24 & 8,81 & 0,92 \\
\hline $\mathrm{A} 600^{\circ} \mathrm{C}$ & 0,22 & 0,41 & 5,13 & 8,75 & 0,88 \\
\hline Masa inicial (g) & 14,744 & 15,711 & 16,287 & 17,454 & 18,372 \\
\hline Masa final $(\mathrm{g})$ & 0,022 & 0,0041 & 0,053 & 0,0875 & 0,0088 \\
\hline
\end{tabular}

Los resultados mostraron que al aumentar el contenido de icopor, aumentó la resistencia térmica. Cabe resaltar que la fibra es necesaria para aumentar la dureza y la resistencia a esfuerzos a los que la pieza está sometida. 


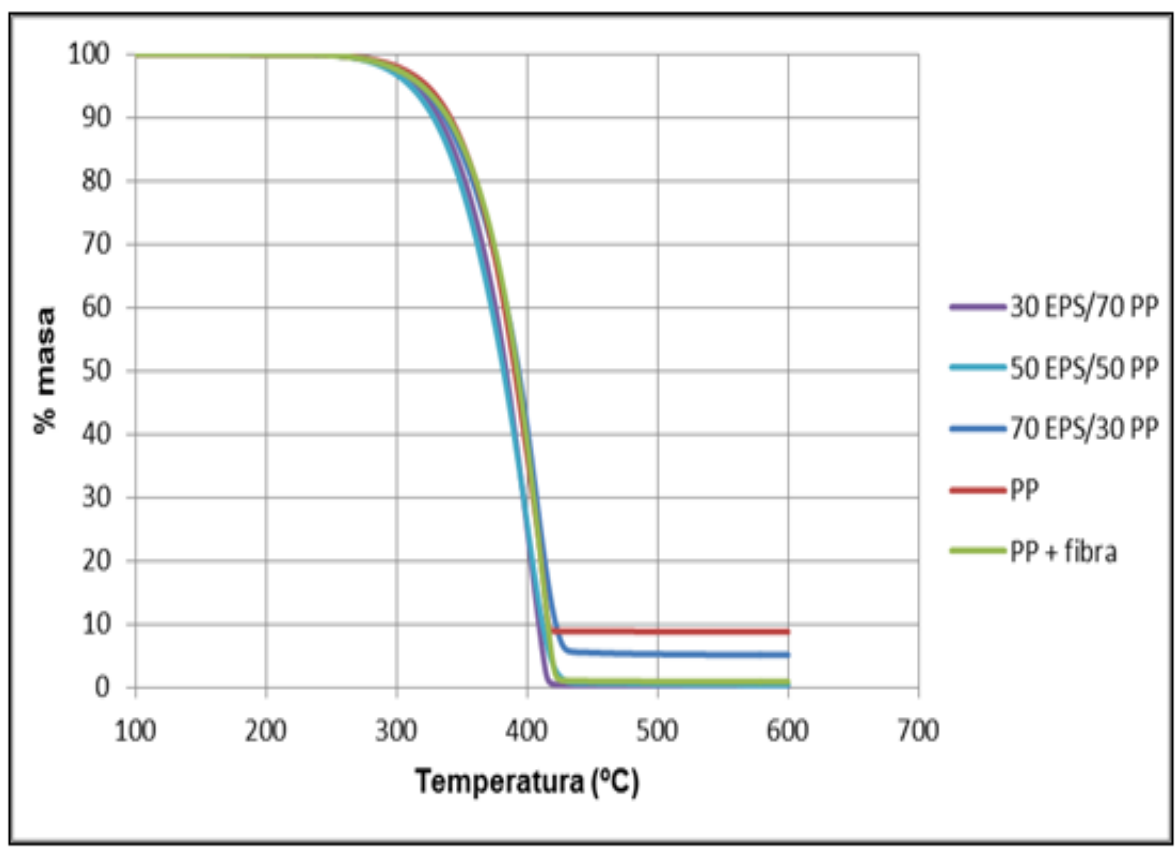

Figura 5. Termograma correspondiente a la composición de la mezcla.

De la misma manera que en el tamaño de partícula, luego de $300^{\circ} \mathrm{C}$ inició una descomposición significativa de la masa de las mezclas de materiales.

Es importante resaltar que se acercó más la variable de composición de la mezcla con el aumento significativo de icopor reciclado al 70\%. Es más recomendable para el análisis térmico manejar proporciones en la mezcla que tamaños de partícula del EPS reciclado.

\section{VALORACIÓN ECONÓMICA}

Para determinar este valor, se calculó la producción de piezas con PP+EPS para 3 años consecutivos plasmados en la Tabla 11. Se tomó una base de 1.000 unidades por mes para un total de 12.000 unidades al año. 
Los flujos de efectivo se calcularon para el año cero teniendo en cuenta una inversión inicial de \$12'000.000 por concepto de adquisición de un molino con cuchillas en el fondo especializado para molienda de materiales livianos como el EPS y $\$ 500.000$ para compra de sacos de empaque del icopor molido.

La tasa de interés mensual para el pago del molino es de $2 \%$ en un total de 36 meses.

Tabla 11. Valor presente neto para 5 años de producción con PP+EPS

\begin{tabular}{|c|c|c|c|c|}
\hline Año & $\begin{array}{l}\text { Producción } \\
\text { (unidades) }\end{array}$ & Flujo de efectivo & Valor presente & $\begin{array}{l}\text { Valor presente neto } \\
\text { (VPN) }\end{array}$ \\
\hline 0 & 1.2000 & $\$-12^{\prime} 500.000$ & $\$-12 ' 500.000,00$ & \multirow{4}{*}{$\$ 27^{\prime} 205.313,7$} \\
\hline 1 & 1.2000 & $\$ 20^{\prime} 040.000$ & $\$ 16^{\prime} 161.290,32$ & \\
\hline 2 & 1.2000 & $\$ 20^{\prime} 040.000$ & $\$ 13^{\prime} 033.298,65$ & \\
\hline 3 & 1.2000 & $\$ 20^{\prime} 040.000$ & $\$ 10^{\prime} 510.724,72$ & \\
\hline
\end{tabular}

El resultado del VPN demuestra que es un proyecto rentable desde el punto de vista económico y financiero, pues la inversión es posible de recuperar en el periodo o año 1 y además permitirá obtener ganancias.

Otra herramienta utilizada fue la tasa interna de retorno (TIR), conocida como la tasa de rentabilidad producto de la reinversión de los flujos de efectivo dentro de la operación del negocio y se expresa en porcentaje (Vaquiro, 2012). En el caso de este estudio, la TIR arroja un valor de $150 \%$ obtenido a partir de los flujos de efectivo y comprobado al reemplazar la tasa de interés por este porcentaje donde el VPN se hace cero. 


\section{CONCLUSIONES}

Aunque existe una disminución en las propiedades de tensión-deformación, es importante resaltar que en cuanto a dureza la mezcla propuesta mejora, y frente a la resistencia térmica no existen cambios significativos en la estabilidad del material. El estudio permitió conocer la posibilidad de reemplazar la fibra de vidrio por icopor como aprovechamiento del residuo, además, económicamente hablando, la tasa interna de retorno es de $150 \%$ a tres años con el cambio de insumos, lo que hace que la investigación acerca de las propiedades del material propuesto sea atractiva. Es recomendable continuar con la investigación sobre este estudio, debido a que se abre un sinnúmero de posibilidades frente a nuevos materiales de fabricación en la industria de transformación de plásticos. Con otro tipo de pruebas, puede determinarse completamente la ficha técnica de la mezcla más adecuada para elaborar un producto terminado con dichas especificaciones.

\section{AGRADECIMIENTOS}

A Ventiladores G.B.A., por permitirnos usar sus equipos industriales para la obtención de las muestras piloto de los materiales.

\section{POTENCIAL CONFLICTO DE INTERESES}

Nos acogemos a las normas colombianas para derechos de autor. El desarrollo de esta investigación tiene como fin favorecer las funciones de proyección social, docencia e investigación en la Universidad Santo Tomás, así como brindar apoyo 
investigativo a los procesos que se llevan a cabo en la empresa Ventiladores G.B.A.

\section{FUENTES DE FINANCIACIÓN}

Recursos propios con apoyo de la Universidad Santo Tomás y la empresa Ventiladores G.B.A.

\section{REFERENCIAS}

- ANAPE. (2011). Propiedades del EPS. Recuperado de http://www.anape.es/pdf/ficha73.pdf

- Bengtsson, M., Le Baillif, M., \& Oksman, K. (2007). Extrusion and mechanical properties of highly filled cellulose fibre-polypropylene composites. Composites Part A: Applied Science and Manufacturing, 38(8), 1922-1931.

- Budynas, R., \& Nisbett, K. (2011). Diseño en ingeniería Mecánica de Shigley. México: McGraw-Hill.

- Cárdenas, J., \& Fonseca, E. (2009). Modelación del comportamiento reológico de asfalto convencional y modificado con polímero reciclado, estudiada desde la 
relación viscosidad- temperatura. Revista EIA, 12, 125-137. Recuperado de http://www.scielo.org.co/pdf/eia/n12/n12a10.pdf

- CEMPRE Colombia. (2013). Guía Técnica Colombiana GTC 53-2. Recuperado de http://www.cempre.org.co

- Contreras, C. (2006). Manejo integral de aspectos ambientales - Residuos sólidos. Recuperado de http://www.javeriana.edu.co/ier/recursos_user/IER/ documentos/OTROS/Pres_Residuos_CamiloC.pdf

- Contreras, M. (2014, 16 de diciembre). Industria de Plásticos en Bogotá. Invest in Bogotá. Recuperado de http://es.investinbogota.org/invierta-enbogota/invertir-bogota/industriales-bogota/industria-plasticos-bogota

- Figueroa Infante, A. S., Reyes Lozano, F. A., Hernández Barrera, D., Jiménez, Ch., \& Bohórquez, N. (2007). Análisis de un asfalto modificado con icopor y su incidencia es una mezcla asfáltica densa en caliente. Ingeniería e Investigación, 27(3), 5-15.

- FRITSCH. (2014). Molinos a cuchillas. Recuperado de www.fritsch.es/productos/molienda/molinos-a-cuchillas/

- Fundación Verde Natura. (2015). Proceso de reciclaje. Recuperado de http://fundacionverdenatura.org/Quienes_Somos.html

- Líbano, E., Visconte, L., \& Pacheco, É. (2012). Propiedades térmicas de compósitos de polipropileno e bentonita organofílica. Polímeros, 22(5), 430435.

- Matheus , P., Dettenborn, J., Zeni, M., \& Zattera, A. (2011). Characterization of composites based on expanded polystyrene wastes and wood flour. Waste Management, 31(4), 779-784. 
- MinCIT -Ministerio de Comercio, Industria y Turismo-. (2013). Informe de industria. Enero de 2013. Colombia. Recuperado de http://www.google.com/url?sa=t\&rct=i\&q=\&esrc=s \&source=web\&cd $=4 \&$ ved $=0$ CC 4 QFjAD\&url=http $\% 3 A \% 2 F \% 2$ Fwww.mincit.gov.co\%2Fdescargar.php\%3Fid\%3D65998 \&ei=MWwJVfnYMlqYNtrUgtgK\&usg=AFQjCNH-moPEO1PPX8 EqHVK6Y8mmlpOjbA\&bvm=bv.88198703,d.eXY

- Plasticbages Industrial, S.L. (2009). Características técnicas del polipropileno. Recuperado de http://www.plasticbages.com/caracteristicaspolipropileno.html

-Valea, A., Juanes, F. J., Miguez, J. C., \& González, M. L. (2008). Aleaciones de polipropileno PP y polietilentereftalato PET reciclados y estudio de sus propiedades.Anales de Mecánica de la Fractura, 2(25), 748-752.

- Vaquiro, J. D. (2012). Tasa interna de retorno - Tir. Pymes Futuro. Recuperado de http://www.pymesfuturo.com/tiretorno.htm

1. Ingeniera Ambiental. Universidad Santo Tomás. Bogotá, Colombia.daylinbetancourt@usantotomas.edu.co. ORCID: 0000-0003-4335-6392

2. Máster en Tecnología Ambiental-UNIA. Universidad Santo Tomás. Bogotá, Colombia.johannasolano@usantotomas.edu.co. ORCID: 0000-0003-4376-5938

Para citar este artículo: Betancourt-S., D. J., \& Solano-M., J. K. (2016). Síntesis y caracterización de la mezcla polipropileno-poliestireno expandido (icopor) reciclado como alternativa para el proceso de producción de autopartes. Revista Luna Azul, 43, 286-310. Recuperado de http://200.21.104.25/lunazul/index.php?option=com_content\&view=article\&id=202

Esta obra está bajo una Licencia de Creative Commons Reconocimiento CC BY

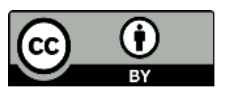

